\title{
The Dutch Open Telescope on La Palma
}

\section{R.J. Rutten, F.C.M. Bettonvil, R.H. Hammerschlag, A. P. L. Jägers, J. Leenaarts, F. Snik, P. Sütterlin, K. Tziotziou, A.G. de Wijn}

Sterrekundig Instituut, Faculteit Natuur- en Sterrenkunde, Utrecht University Postbus 80000 , NL-3508 TA Utrecht, The Netherlands

email: R.J.Rutten@astro.uu.nl

\begin{abstract}
The Dutch Open Telescope (DOT) on La Palma is an innovative solar telescope combining open telescope structure and an open support tower with a multi-wavelength imaging assembly and with synchronous speckle cameras to generate high-resolution movies which sample different layers of the solar atmosphere simultaneously and co-spatially at high resolution over long durations. The DOT test and development phase is nearly concluded. The installation of an advanced speckle processor enables full science utilization including "Open-DOT" time allocation to the international community. Co-pointing with spectropolarimeters at other Canary Island telescopes and with TRACE furnishes valuable Solar-B precursor capabilities.
\end{abstract}

\section{Introduction}

Solar physics is on the upswing worldwide. Three revolutionary developments are behind this blossoming: (i) continuous monitoring from space, in particular with SOHO, (ii) the development of effective wavefront restoration techniques for groundbased optical telescopes, and (iii) the advent of numerical modelling at a level of complexity commensurate with solar reality. In each of these revolutions, solar physics evolves from static snapshot sampling to dynamic time-sequence analysis. This is important because most if not all of the abundant complex phenomena which our star displays so gracefully to teach us astrophysics are inherently dynamic. For example, the EUV movies from EIT and TRACE demonstrate unequivocally that coronal glimpsing at rare solar eclipses is not the way to diagnose coronal physics. Solar physics becomes a science of movies a step beyond the trend of general astronomy to become a science of pictures. Indeed, many solar "Astronomy picture of the day" entries link to movies.

The Dutch Open Telescope (DOT) described here shares in the second revolution: it is a groundbased movie maker. The DOT produces long-duration image sequences at sustained high angular resolution which sample the solar atmosphere tomographically at different wavelengths, synchronously and co-spatially. This conference on multi-wavelength investigations of solar activity happens to coincide with the DOT's coming of age in this field, presently transiting from tests and instrumentation development to full science utilization. The DOT indeed suits very well to multi-wavelength studies of many aspects of solar activity. This paper gives a summary. More information is found in Rutten et al. (2004a) and at URL http://dot.astro.uu.nl/ where one may also download DOT images and movies and find instructions on how to obtain DOT data and observing time.

\section{Open principle}

At its conception by Hammerschlag at the instigation of the late C. Zwaan, the DOT was designed as site verification telescope for LEST, the European solar telescope which 

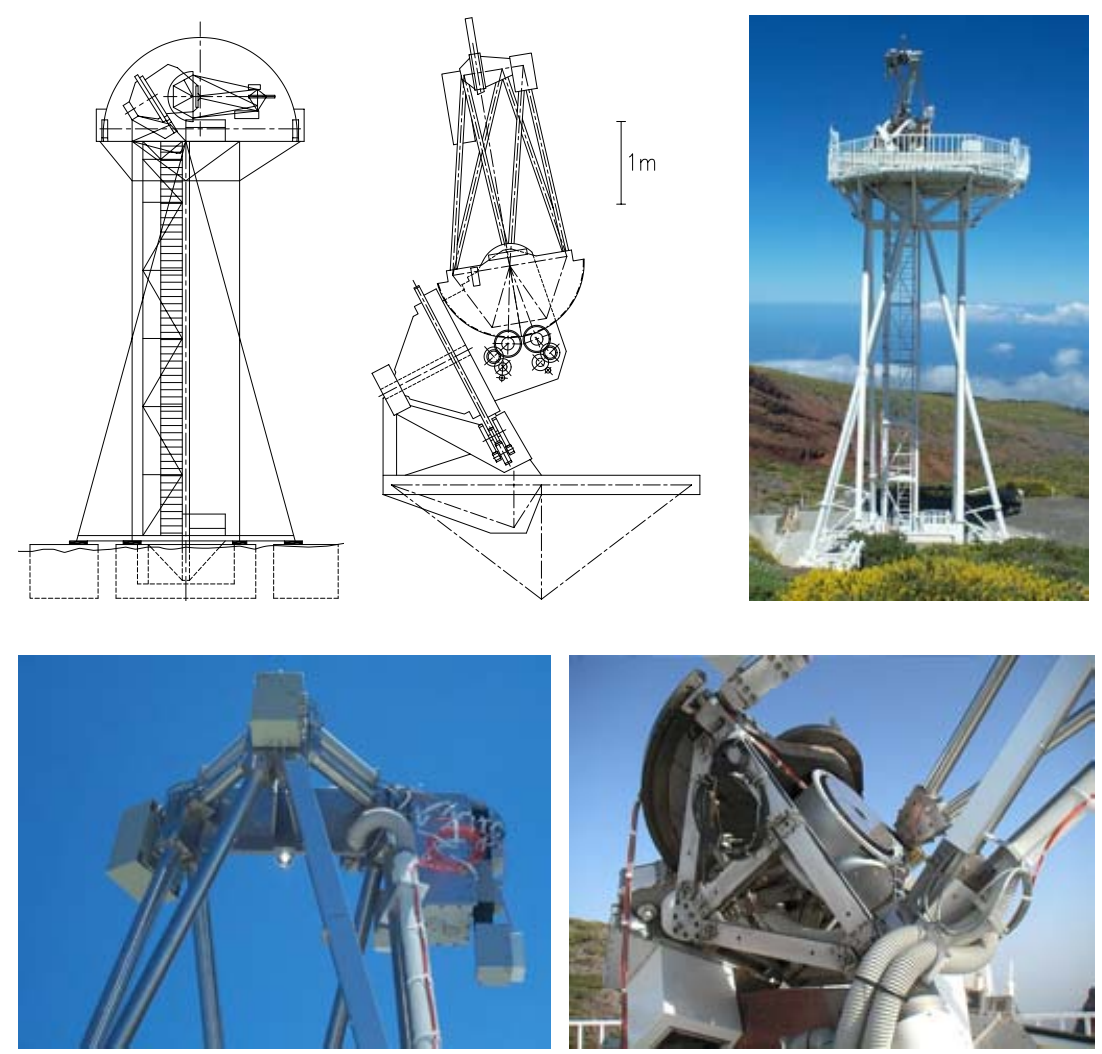

Figure 1. Clockwise: structural DOT sketches, DOT, primary mirror in parallactic mount, telescope top with prime-focus heat stop and post-focus equipment. The mirror diameter is $45 \mathrm{~cm}$ but the mount can accommodate a $140 \mathrm{~cm}$ mirror. The flexible tubes serve for air suction around prime focus. The reflective heat stop is watercooled.

never materialized. Over the years the DOT grew into a solar telescope per sé and eventually made it to La Palma where it now stands close to the Swedish Solar 1-m Telescope (SST) from whose building it is operated. The SST is a closed refractor relying on evacuation to avoid internal telescope seeing and represents the aperture limit feasible with evacuation. In utter contrast, the DOT is an open reflector and the pathfinder towards larger diameter.

The DOT's open principle was formulated already in the early 1970s when Zwaan and Hammerschlag participated in JOSO's extensive site tests that led to the identification of the Canary Island volcanos as primary choice for European solar telescopes (Brandt \& Righini 1985, cf. Rutten 2000). The design was described by Hammerschlag (1973, 1981, 1983). Further technical detail is given in Hammerschlag \& Bettonvil (1998), Hammerschlag et al. (2003) and Bettonvil et al. (2003).

The realization took three decades mostly due to lack of manpower and funding (but note that if the project had been larger it would probably not have survived the deteriorating university climate for instrumentation in those years). Amazingly, the DOT remains innovative and highly competitive even after so many years, while its demonstration of the open principle came just in time for the large solar telescope projects that are now underway elsewhere. That demonstration culminated in the beautiful DOT sunspot movie posted as "Astronomy picture of the day" on February 23, 2000 and suggested that non-evacuation design may be combined with the advent of adaptive optics to aim 
for angular resolution beyond one-meter aperture to reach new vistas in solar scrutiny. The spectacular images from the SST confirm that new realms of solar physics open already at 0.1 arcsec resolution. The current post-LEST beyond-one-meter solar telescope projects, i.e., the $1.5 \mathrm{~m}$ German GREGOR (http://gregor.kis.uni-freiburg.de), the $1.6 \mathrm{~m} \mathrm{NST} \mathrm{for} \mathrm{Big} \mathrm{Bear} \mathrm{(http://www.bbso.njit.edu)} \mathrm{and} \mathrm{particularly} \mathrm{the} 4 \mathrm{~m}$ ATST (http://atst.nso.edu; see also S.L. Keil's preceding paper in this volume) indeed combine non-evacuation with adaptive optics.

Various features contribute to the DOT's success as open telescope. First of all the site characteristics. The Northern trade winds blowing upslope over the Roque de los Muchachos site at La Palma tend to be strong and fairly laminar; these bring the best local seeing and at the same time flush our telescope. Sites without such winds require elaborate active mirror venting. Secondly, the DOT tower is also open and the telescope mount lifts the primary mirror well above the open-mesh platform including the rim presented by the folded-down canopy. More massive buildings, towers, and platforms are likely to cause more local turbulence. The SST tower is closed, but it is tall and slender and also the SST lifts its exposed primary imaging element (the singlet objective doubling as vacuum entrance window) high above its partially open platform. The high seeing conformity between the DOT and the SST indicates that both towers perform well. Thirdly, much DOT design ingenuity and construction effort has gone into avoiding wind-induced platform and telescope shake. Fear of the latter is a major motivation for the complex ventilated enclosure in the current ATST design.

\section{Speckle tomography}

After the installation of the DOT on La Palma and the initial verification of the viability of the open principle and of the excellent DOT pointing stability with a simple video camera, we obtained funding to turn the DOT into a multi-wavelength tomographic imager. This choice was suggested by our limited manpower, modest means, and the inaccessibility of the secondary optics in the telescope top compared to the optics laboratories available for instrument set-up at e.g., the SST and the Dunn Solar Telescope. In addition, we proposed to employ speckle reconstruction as the primary means to remove remaining wavefront perturbations, in the hopeful expectation that the general computer advance would permit us to progress to large-volume speckle processing within a few years.

These choices defined the development of an elaborate multi-wavelength post-focus reimaging system feeding multiple speckle cameras through appropriate filters. The latter are specified in Table 1. Each filter required its own re-imaging optics train with optimum aberration correction. Extensive on-telescope tests were performed to ensure best optics performance. Many movies presently available on the DOT website resulted from such tests. In the meantime, the four re-imaging channels containing interference filters have been completed and are functioning. The more recent "quartet" movies on the DOT website are synchronous and co-spatial at all four wavelengths.

The images in the different wavelength channels are registered with identical Hitachi KP-F100 cameras with Sony ICX085 CCD chips, $1296 \times 1030 \mathrm{px}^{2}$, pixel size $6.7 \mu \mathrm{m}$ square, well depth 16000 electrons, 10-bit readout, up to $12 \mathrm{f} / \mathrm{s}$ frame rate. Affordability dictated the selection of these machine-vision cameras, which pose linearity and noise problems that cannot be fully corrected in the reduction. Their chip size limits the field to $92 \times 73 \operatorname{arcsec}^{2}$ at 0.071 arcsec/px sampling. A few arcseconds are usually lost to image motion, residual solar rotation and guider flexure, differential refraction, activeregion proper motion, and movie co-alignment. Each camera is connected through a dual 


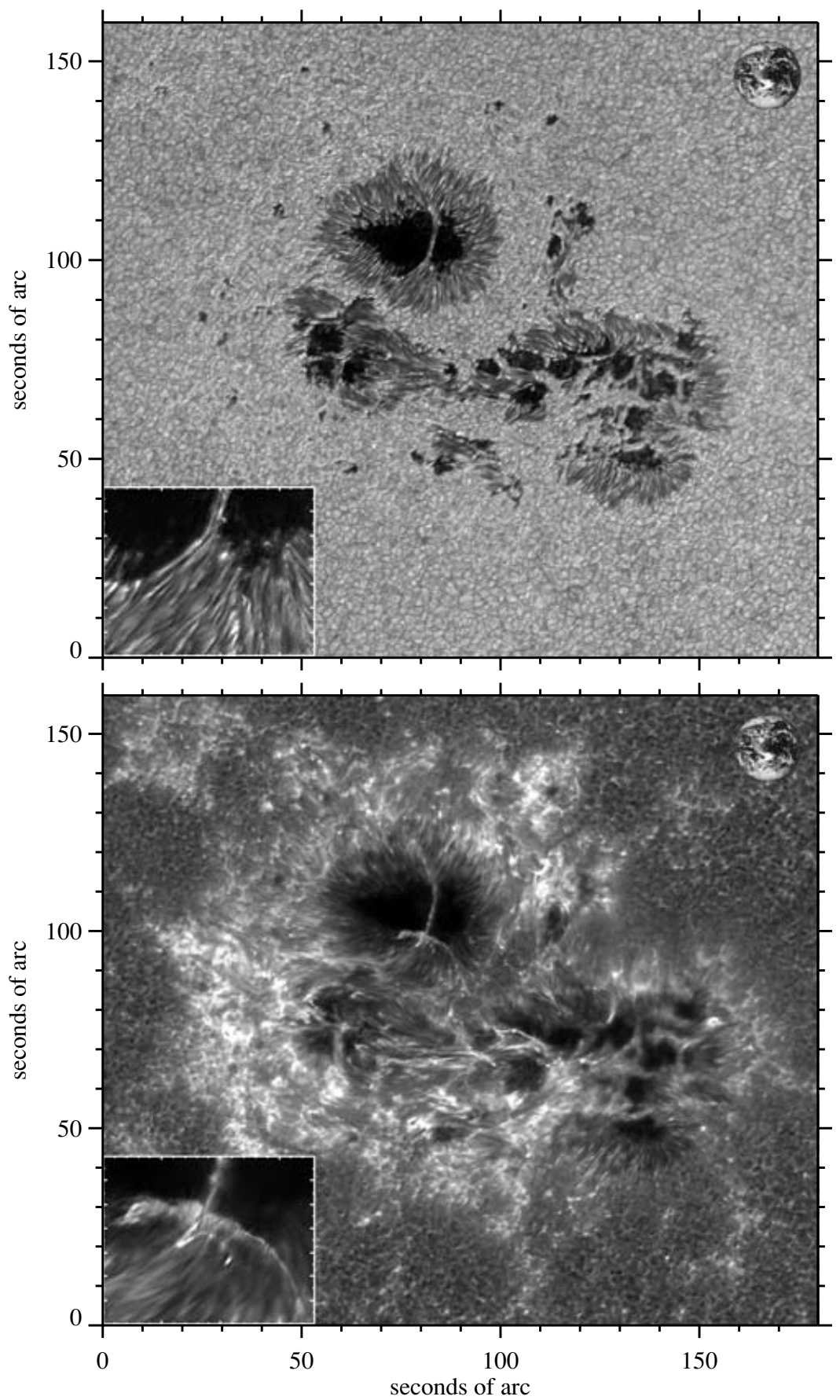

Figure 2. Co-spatial and synchronous speckle-reconstructed DOT image mosaics of solar active region AR10375 taken on June 6, 2003. The Earth inserts indicate the geometric scale. The lower-left inserts show a magnified part of the largest spot. Top: G band, consisting of molecular $\mathrm{CH}$ lines around $430.5 \mathrm{~nm}$. It shows a large active region with multiple sunspots, surrounded by solar granulation and tiny bright magnetic elements within the intergranular lanes. Bottom: overlying chromosphere about $500 \mathrm{~km}$ higher up in Ca II H $(396.8 \mathrm{~nm})$. The angular resolution in the original images is close to the 0.2 arcsec DOT diffraction limit over the whole field. 
Table 1. DOT filter specification. The $6563 \AA$ and $3968 \AA$ interference filters can be tilted under computer control to shift their passbands blueward from the $\mathrm{H} \alpha$ and $\mathrm{Ca}$ II $\mathrm{H}$ line centers, respectively. The two Lyot filters can be stepped through $\mathrm{H} \alpha$ and $\mathrm{Ba}$ II 4554 , respectively, to produce Dopplergrams. Polarimetry with the Ba II 4554 filter is under study.

\begin{tabular}{lcccc}
\hline designation & $\lambda(\AA)$ & width $(\AA)$ & type & tuning \\
\hline blue continuum & 4320 & 6 & interference & fixed \\
red continuum & 6563 & 3 & interference & tiltable \\
G band & 4305 & 10 & interference & fixed \\
Ca II H & 3968 & 1.35 & interference & tiltable \\
H $\alpha$ & 6563 & 0.25 & Lyot & tunable \\
Ba II & 4554 & 0.08 & Lyot & tunable \\
\hline
\end{tabular}

400-Mbps 100-m long optical fiber link to its own data-acquisition computer in the SST building.

The tunable Lyot filter for $\mathrm{H} \alpha$ was formerly in use at ORSO (Gaizauskas 1976) and is presently being installed. The final channel will harbour the tunable Lyot filter for Ba II 4554 built at the Irkutsk Institute of Solar-Terrestrial Physics by V.I. Skomorovsky and coworkers. Its outstanding Dopplergram capability was demonstrated with the former Swedish Vacuum Solar Telescope (Sütterlin et al. 2001, Kushtal \& Skomorovsky 2002). Its installation is planned for next spring. A Stokes- $V$ magnetometry mode is under study.

The wavelength setting of the shiftable and tunable filters can be changed between successive speckle bursts under program control. In the extended Ca II H wing such stepping represents shifting an LTE brightness diagnostic in height of formation. One may choose to take successive bursts in alternating Ca II H wing locations to vary the height of response to temperature fluctuations. The red interference filter usually samples the continuum at $6540 \AA$, but it can also be used to register the profile-integrated $\mathrm{H} \alpha$ emission from an off-limb prominence.

The telescope focus is maintained with a phase-diverse imaging system using a video camera and frame grabber to monitor the granulation contrast in two image planes. Balancing the two measured contrasts defines the location of the focal plane as midway between the two samplings.

Speckle registration was started at the DOT by Sütterlin building on the Göttingen code. All movies available on the DOT website are speckle-restored. An important advantage of this image-plane technique is that the whole field of view gets corrected since it is split into about a thousand subfields, each smaller than the isoplanatic patch, which are re-merged after individual speckle reconstruction.

A major disadvantage of the DOT speckle reconstruction sofar was the severe bottleneck which it imposed on our movie production. However, our optimistic expectation that high-volume speckle reconstruction should become affordable has indeed materialized: we are presently installing a sophisticated 70-Xeon processor farm on La Palma which is designed to process a full 1.8 Terabyte run (6 cameras speckle-bursting continuously during eight hours) within one day. An elaborate cooling system stores the generated heat for release around sunset and sunrise. 

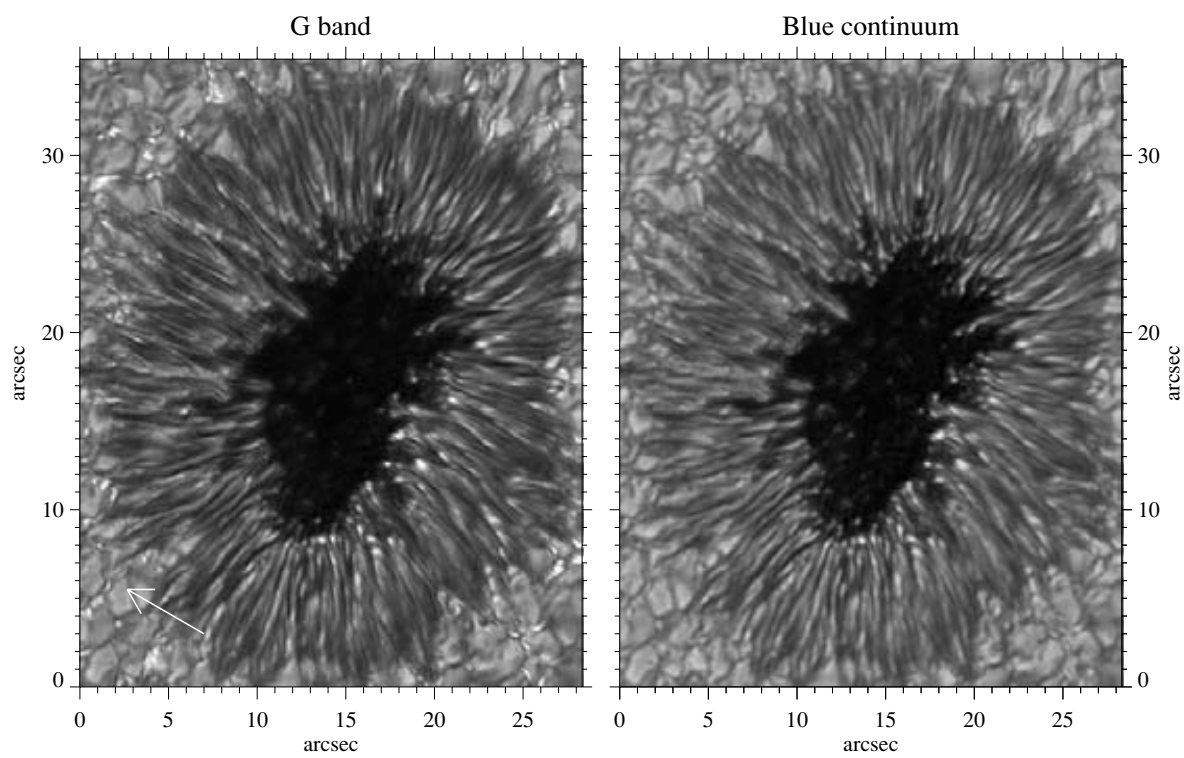

Figure 3. Subfield of synchronous sunspot (NOAA 10425) images taken on August 9, 2003. Left: G band. Right: blue continuum. The arrow marks the direction to disk center. The penumbral filaments display dark cores, confirming the SST discovery of Scharmer et al. (2002) and demonstrating that off-center spots show them more distinctly on the disk-center side. From Sütterlin et al. (2004).

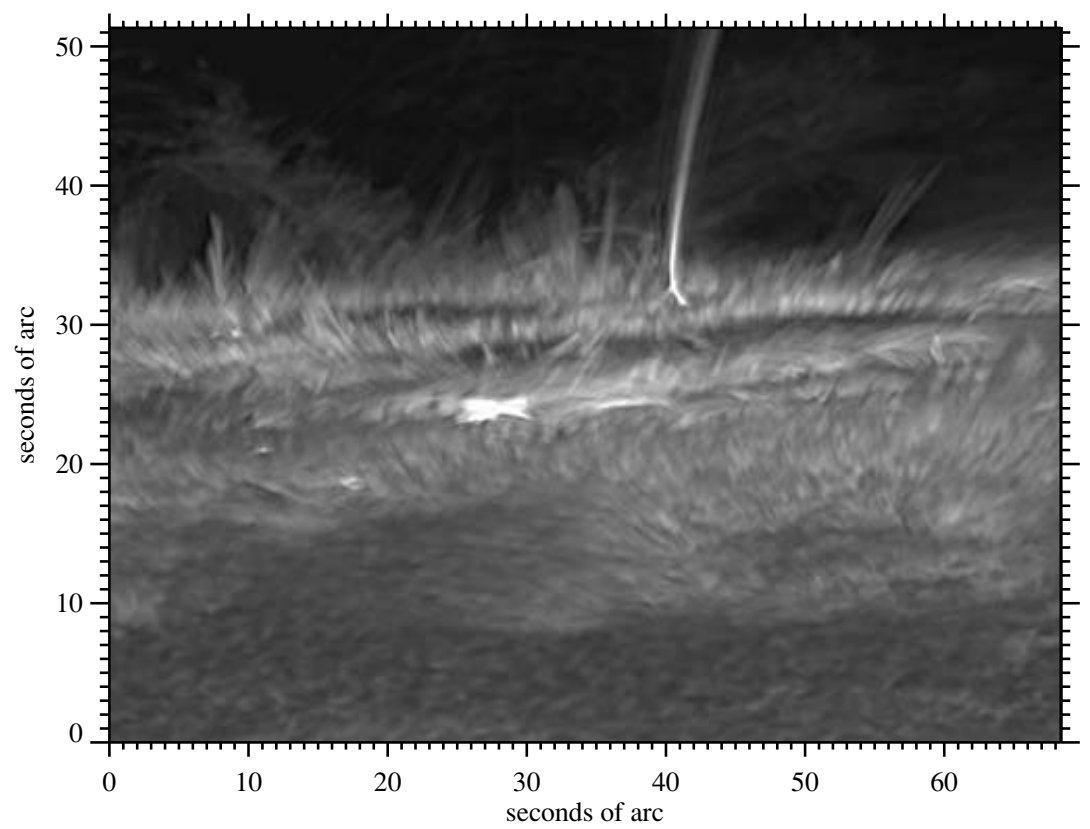

Figure 4. Active region AR10486 at the solar limb on November 4, 2003. This Ca II H image was taken just before sunset, a few hours before the most intense X-ray flare sofar recorded. The image vividly demonstrates that the solar chromosphere consists of a highly structured dynamical forest of spicules/fibrils/mottles which is ill-described by horizontally homogeneous "standard" models. The bright surge appears dark in TRACE $195 \AA$ and shows supersonic upward feature motions and $400 \mathrm{~s}$ brightness oscillation (de Wijn et al., in preparation). 


\section{Test-phase results}

Some of the DOT movies produced during the development and test phase have been used for initial research. Sütterlin (2001), Sobotka \& Sütterlin (2001) and Sütterlin et al. (2004) analysed penumbral fine structure (cf. Fig. 3). Rouppe van der Voort (2003) studied the penumbral Evershed flow and Rouppe van der Voort et al. (2003) analysed umbral flashes. Nisenson et al. (2003) measured motions of magnetic elements observed as G-band bright points in quiet-sun areas, while Bovelet \& Wiehr (2003) did so for the magnetic elements appearing in sunspot moats. Rutten et al. (2004a) compared the reversed granulation observed in the wing of $\mathrm{Ca}$ II $\mathrm{H}$ with the underlying granulation; their observations are presently being compared with radiation-hydrodynamics simulation results by Leenaarts \& Wedemeyer-Böhm.

\section{Open-DOT program}

The DOT now transits from testbed to science facility. The multi-wavelength system makes the DOT a high-resolution mapper of solar-atmosphere topology and dynamics in tomographic fashion. The high-speed speckle processor enables production of specklereconstructed movies in far greater number than before. The DOT standard product will consist of co-spatial synchronous image sequences taken at the wavelengths of Table 1 during multiple hours at 20-30 second speckle burst sampling cadence. The field of view covers up to $90 \times 70$ arcsec at 0.071 arcsec pixel resolution. There is no diurnal image rotation. The guiding may or may not compensate for solar rotation, as desired. Such high-resolution long-duration multi-camera sequences from the DOT provide excellent science input in themselves, but are also highly valuable as context tomography for smaller-field spectrometry and polarimetry at other telescopes, and when combined with coronal EUV imaging from space.

DOT data can be delivered whenever the DOT is manned and the seeing is reasonable. At Fried parameter $10 \mathrm{~cm}$ (good but not perfect) the resolution already approaches the 0.2 arcsec diffraction limit. This happens not infrequently during multiple hours. Manning the DOT is limited by the small size of our team. We hope to operate the DOT during most of the summer months when good seeing occurs frequently, and in specific 1-2 week campaigns the rest of the year. External usage may employ service-mode observing in which the DOT team operates the telescope, and common-user-mode observing in which a proposer participates on-site in telescope operation, target selection, and pointing. These modes make up our "Open-DOT" program. In addition, all DOT data are public. The DOT movies will be supplied on DVDs awaiting Virtual Solar Observatory access at sufficient bandwidth.

It is our intention to schedule Open-DOT observing time on the basis of scientific merit. It is likely that preference will often be given to programs proposing co-pointing of the DOT with the SST and/or TRACE to exploit the complementary science capabilities of these telescopes. In particular, DOT speckle tomography will combine most fruitfully with adaptive-optics spectrometry and polarimetry at the SST and with EUV coronal imaging by TRACE. Important boundary conditions are that the DOT and SST share virtually identical seeing while TRACE performs predictably, that the DOT and SST control rooms are adjacent in the SST building, and that precise inter-telescope co-registration is very well feasible through overlapping Ca II H \& K and/or continuum imaging (ultraviolet and/or white light, respectively, for TRACE).

Co-pointing the DOT, the SST and TRACE may deliver Solar-B-like data sequences with similar angular resolution over multiple-hour durations. From late 2006 onward 
Solar-B itself should furnish much longer sequences without limitation by the terrestrial weather and day/night cycle, but it is important to start now already on Solar-B science using the medium-duration sequences at Solar-B resolution which now become feasible thanks to groundbased wavefront restoration. We welcome proposals for such research.

\section{Acknowledgements}

The DOT is operated by Utrecht University at the Spanish Observatorio del Roque de los Muchachos of the Instituto de Astrofísica de Canarias from the SST building of the Institute for Solar Physics of the Royal Swedish Academy of Sciences. The DOT efforts are funded by Utrecht University, the Netherlands Organisation for Scientific Research NWO, the Netherlands Graduate School for Astronomy NOVA, and SOZOU. The DOT was built by the workshops of Utrecht University and the Central Workshop of Delft University of Technology with funding from Technology Foundation STW. The speckle data acquisition and re-imaging systems were built by the Instrumentele Groep Fysica at Utrecht. The Netherlands Foundation for Research in Astronomy ASTRON provides much assistance. The DOT speckle processor is funded by NWO. Adaptation of the Göttingen speckle code was part of the EC-TMR European Solar Magnetometry Network ESMN. The present DOT efforts are part of the EC-RTN European Solar Magnetism Network under contract HPRN-CT-2002-00313. R.J. Rutten and P. Sütterlin acknowledge support from the Leids Kerkhoven-Bosscha Fonds to participate in this conference and thank its organizers for a very good meeting.

\section{References}

Bettonvil, F. C. M., Hammerschlag, R. H., Sütterlin, P., Jägers, A. P., \& Rutten, R. J. 2003, Procs. SPIE 4853, 306

Bovelet, B. \& Wiehr, E. 2003, A\&A, 412, 249

Brandt, P. N. \& Righini, A. 1985, Vistas in Astronomy, 28, 437

Gaizauskas, V. 1976, JRAS Can., 70, 1

Hammerschlag, R. H. 1973, JOSO Annual Report, 85

Hammerschlag, R. H. 1981, in Solar Instrumentation: What's Next?, ed. R. B. Dunn, Proc. Sacramento Peak National Observatory Conference, Sunspot, New Mexico, 547

Hammerschlag, R. H. 1983, Procs. SPIE, 444, 138

Hammerschlag, R. H. \& Bettonvil, F. C. M. 1998, New Astronomy Reviews, 42, 485

Hammerschlag, R. H., Jägers, A. P., \& Bettonvil, F. C. M. 2003, Procs. SPIE 4853, 294

Kushtal, G. I. \& Skomorovsky, V. I. 2002, Procs. SPIE, 4900, 504

Nisenson, P., van Ballegooijen, A. A., de Wijn, A. G., \& Sütterlin, P. 2003, ApJ, 587, 458

Rouppe van der Voort, L. H. M. 2003, A\&A, 397, 757

Rouppe van der Voort, L. H. M., Rutten, R. J., Sütterlin, P., Sloover, P. J., \& Krijger, J. M. 2003, A\&A, 403, 277

Rutten, R. J. 2000, JOSO Annual Report 1999, 4

Rutten, R. J., Hammerschlag, R. H., Bettonvil, F. C. M., Sütterlin, P., \& de Wijn, A. G. 2004a, A\&A, 413, 1183

Rutten, R. J., de Wijn, A. G., \& Sütterlin, P. 2004b, A\&A, 416, 333

Scharmer, G. B., Gudiksen, B. V., Kiselman, D., Löfdahl, M. G., \& Rouppe van der Voort, L. H. M. 2002, Nature 420, 151

Sobotka, M. \& Sütterlin, P. 2001, A\&A, 380, 714

Sütterlin, P. 2001, A\&A, 374, 21

Sütterlin, P., Rubio, L. R. B., \& Schlichenmaier, R. 2004, A\&A, in press

Sütterlin, P., Rutten, R. J., \& Skomorovsky, V. I. 2001, A\&A, 378, 251 